\title{
A Neutrosophic Image Retrieval Classifier
}

\author{
A. A. Salama \\ Port Said University, Faculty of \\ Science, \\ Department of Mathematics \\ and Computer Science
}

\author{
Mohamed Eisa \\ Port Said University, Higher \\ Institute of Management and \\ Computer, Department of \\ Computer Science
}

\author{
A. E. Fawzy \\ Port Said University, Higher \\ Institute of Management and \\ Computer, Department of \\ Computer Science
}

\begin{abstract}
In this paper, we propose a two-phase Content-Based Retrieval System for images embedded in the Neutrosophic domain. In this first phase, we extract a set of features to represent the content of each image in the training database. In the second phase, a similarity measurement is used to determine the distance between the image under consideration (query image), and each image in the training database, using their feature vectors constructed in the first phase. Hence, the $\mathrm{N}$ most similar images are retrieved.
\end{abstract}

\section{Keywords}

Images in the Neutrosophic Domain, similarity measures, Euclidean distances.

\section{INTRODUCTION}

Because of a huge growth of digital images, Content Based Image Retrieval (CBIR) is a wide research area searching for images from a large database using visual information which based on a given query image [13]. The Content Based Image Retrieval (CBIR)[3]goalis to retrieve images relevant to a query images which selected by a user. The image in CBIR is described by extracted low-level visual features, such as color, texture and shape $[9,14,17]$. When a user selscts one or more query images, the criteria of this image description ranks the images of an image database according to their similarity with the query images. Finally, the most similar images are retrieved. The feature extraction aim is to create high-level data (pixel values). The visual features can be ordered in three levels: low level features (primitive), middle level features (logical) and high level features (abstract). Most of recently systems were depended on low level features (color, shape). But both of mid-level and high-level image representations are now in demand. The CBIR systemefficiencydepends on extracted features [8]. The first and the main task in the CBIR systems to retrieve the similar images from database is Feature extraction. Then, using similarity measurement to decide how close an image is to another image. The selected similarity metrics impact on the performance of content-based image retrieval. The feature vectors selected type, determines the measurement type that used to compare their similarity. The most common metric which used to measure the distance between two points in multi-dimensional space is Euclidean distance. The proposed work primarily focuses on using Neutrosophic Sets in Image Retrieval system. The Neutrosophic logic was proposed in 1995, 1998, 1999 and 2002,by smarandache[25-28] that define the notion of Neutrosophic Set, which is a generalized of Zadeh's fuzzy set and Atanassov's intuitionistic fuzzy set. TheNeutrosophic concepts which are the degree of membership (T), Indeterminacy (I) and the degree of non-membership (F) of each element have been investigated by Salama et al. [6,1922].

\section{IMAGES IN THE NEUTROSOPHIC DOMAIN}

Let $\mathrm{U}$ be a universe of discourse and $\mathrm{W}$ is a set in $\mathrm{U}$ which composed of bright pixels. A Neutrosophic images $\mathrm{P}_{\mathrm{NS}}$ is characterized by three sub sets T, I, and F. that can be defined as $\mathrm{T}$ is the degree of membership, $\mathrm{I}$ is the degree of indeterminacy, and $\mathrm{F}$ is the degree of non-membership. In the image, a pixel $\mathrm{P}$ in the image is described as $\mathrm{P}(\mathrm{T}, \mathrm{I}, \mathrm{F})$ that belongs to $\mathrm{W}$ by its $\mathrm{t} \%$ is true in the bright pixel, $\mathrm{i} \%$ is the indeterminate and $\mathrm{f} \%$ is false where $\mathrm{t}$ varies in $\mathrm{T}$, $\mathrm{i}$ varies in $\mathrm{I}$, and $f$ varies in $F$. The pixelp(i,j)in the image domain, is transformed to $\mathrm{NDP}_{\mathrm{NS}}(\mathrm{i}, \mathrm{j})=\{\mathrm{T}(\mathrm{i}, \mathrm{j}), \mathrm{I}(\mathrm{i}, \mathrm{j}), \mathrm{F}(\mathrm{i}, \mathrm{j})\}$. Where $T(i, j)$ belongs to white set, $I(i, j)$ belongs to indeterminate set and $I(i, j)$ belongs to non-white set.

Which can be defined as [2]:

$$
\begin{aligned}
& P_{N S}(i, j)=\{T(i, j), I(i, j), F(i, j)\} \\
& T(i, j)=\frac{\overline{g(i, j)}-\bar{g}_{\text {min }}}{\bar{g}_{\text {max }}-\bar{g}_{\text {min }}} \\
& I(i, j)=1-\frac{H_{0}(i, j)-H_{0}}{H_{0_{\text {max }}}-H_{0_{\text {min }}}} \\
& F(i, j)=1-T(i, j) \\
& H_{0}(i, j)=a b s(g(i, j)-\overline{g(i, j)})
\end{aligned}
$$

Where $\overline{g(i, j)}$ represents the local mean value of the pixels of window size, and $H_{0}(i, j)$ which can be defined as the homogeneity value of $\mathrm{T}$ at $(\mathrm{i}, \mathrm{j})$, that described by the absolute value of difference between intensity $g(i, j)$ and its local mean value $\overline{g(i, j)}$.

\section{FEATURES EXTRACTION}

The basic task of CBIR is Feature Extraction. Features may be contain both text-based features (key, words, and annotations) and visual features (color, texture, shape, faces). The feature extraction target is to create high-level data (pixel values). The visual features can be ordered in three levels: low level features (primitive), middle level features (logical) and high level features (abstract). Most of recent system was depended on low level features (color, shape) [1]. 


\subsection{Texture Feature for Image Retrieval}

Using the gray level co-occurrence matrix in the texture feature extraction for the query image and the first image in the database to extract the texture feature vector [10]. The cooccurrence matrix representation technique used to give the intensity values and the distribution of the intensities. The selected features used for retrieving texture properties are Energy, Entropy, Inverse difference, Moment of inertia, Mean, Variance, Skewness, Distribution uniformity, Local stationary and Homogeneity [8].

\subsubsection{Neutrosophic Entropy}

The Shannons Entropy is the an absolute limit on the best possible average length of lossless encoding or compression of an information source. Needing $\log _{2}(n)$ bits to represent a variable which can take one of $n$ values if $n$ is a power of 2 . The entropy is equal to the number of bits equality between number of bits and shannons holds only while all outcomes are equally probable, in case of these values are equally probable. The observations of that event is less informative, if one of the events is more probable than others. In the converse way, rare events provide more information when observed. When the observation of less probable events occurs more rarely, the net effect is that the entropy received from nonuniformly distributed data is $\log _{2}(n)$. When one of the outcomes is certain, the entropy is zero. When a probability distribution of the source is known Shannon entropy quantifies all these considerations exactly quantifies. The Entropy can be only takes into account the probability of observing a specific event, so the information that encapsulates is information about the underlying probability distribution, not the meaning of the events themselves [23]. Entropy is defined as [12]:

$$
\text { Entropy }=\sum_{i} \sum_{j} P(i, j) \log P(i, j)
$$

Although, the Neutrosophic Set Entropy was defined in one dimension which presented in [4], We will define it in two dimension to be as follows:

$$
\begin{aligned}
& E n_{N s}=E n_{T}+E n_{I} \\
& +E n_{F} \\
& E n_{T}=\sum_{i} \sum_{j} P_{T}(i, j) \log P_{T}(i, j) \\
& E n_{I}=\sum_{i} \sum_{j} P_{I}(i, j) \log P_{I}(i, j) \\
& E n_{F}=\sum_{i} \sum_{j} P_{F}(i, j) \log P_{F}(i, j)
\end{aligned}
$$

Where $\mathrm{P}$ contains the histogram counts. Because, we used the interval between 0 and $1, \log P(i, j)$ may have negative values. So, we use the absolute of $E n_{T}, E n_{I}, a n d E n_{F}$.

\subsubsection{Neutrosophic Contrast:}

The Contrast describe the difference in luminance or color that makes an object distinguishable. In the real world, contrast is determined by the difference in the color and brightness of the object and other objects within the same field of view. The most sensitive to contrast is the human visual system than absolute luminance. The contrast ratio or dynamic range is the maximum contrast of an image. It is the the measure of the intensity contrast between a pixel and its neighbor over the whole image, it can be defined as [24]:

Contrast $=\sum_{i} \sum_{j}(i-j)^{2} P(i, j)$
We will define the Neutrosophic set Contrast to be as follows:

$$
\begin{aligned}
\text { contrast }_{N S} & =\text { contrast }_{T}+\text { contrast }_{I}+\text { contrast }_{F} \\
\text { contrast }_{T} & =\sum_{i} \sum_{j}(i-j)^{2} P_{T}(i, j) \\
\text { contrast }_{I} & =\sum_{i} \sum_{j}(i-j)^{2} P_{I}(i, j) \\
\text { contrast }_{F} & =\sum_{i} \sum_{j}(i-j)^{2} P_{F}(i, j)
\end{aligned}
$$

\subsubsection{Neutrosophic Energy:}

The energy can be defined as the sum of squared elements Which defined as [7]:

Energy $=\sum_{i} \sum_{j} P^{2}(i, j)$

We will define the Neutrosophic set Energy to be as follows:

$$
\begin{aligned}
& \text { Energy }_{I}=\sum_{i} \sum_{j} P_{T}{ }^{2}(i, j) \\
& \text { Energy }_{I}=\sum_{i} \sum_{j} P_{I}{ }^{2}(i, j) \\
& \text { Energy }_{F}=\sum_{i} \sum_{j} P_{F}{ }^{2}(i, j)
\end{aligned}
$$$$
\operatorname{Energy}_{N S}=\text { Energy }_{T}+\text { Energy }_{I}+\text { Energy }_{F}
$$

\subsubsection{Neutrosophic Homogeneity:}

The properties of a data set, or several datasets called Homogeneity. Homogeneity studied the several degrees of complexity. For example, the homoscedasticity considerations examine how much the variability of data-values changes throughout a dataset. However, homogeneity questions apply to all aspects of the statistical distributions, including the location parameter. Homogeneity is related to the validity of the often convenient assumption that the statistical properties of any one part of an overall dataset are the same as any other part. Homogeneity measures the difference or similarities between the many studies, in meta-analysis, that combines the data from many studies,. That is a value which measures the closeness of the distribution of elements. Which defined as [11]:

$$
\text { Homo }=\sum_{i} \sum_{j} \frac{P(i, j)}{1+|i-j|}
$$

We will define the Neutrosophic set Homogeneity to be as follows:

$$
\begin{aligned}
\mathrm{Homo}_{N S} & =\mathrm{Homo}_{T}+\mathrm{Homo}_{I}+\mathrm{Homo}_{F} \\
\mathrm{Homo}_{T} & =\sum_{i} \sum_{j} \frac{P_{T}(i, j)}{1+|i-j|} \\
\mathrm{Homo}_{I} & =\sum_{i} \sum_{j} \frac{P_{I}(i, j)}{1+|i-j|} \\
\mathrm{Homo}_{F} & =\sum_{i} \sum_{j} \frac{P_{F}(i, j)}{1+|i-j|}
\end{aligned}
$$

Recently, the Euclidean distance is calculated the distance between the query image and the first image in the database and stored in an array. This process is repeated for the remaining images in the database followed by storing their values respectively. The array is stored now in ascending order and displayed the first 9 closest matches. 


\section{NEUTROSOPHIC EUCLIDEAN DISTANCE}

Similarity measuresplay important role in Image Retrieval, that is for Content Based Image Retrieval calculates visual similarity between a query image and images in a database. Therefore, the retrieval result is not a single image but a number of images ranked by their similarities with the query image.The query image will be more similar to the database images if the distance is smaller. Different similarity measures will affect retrieval performances of an image retrieval system significantly so, it is important to find best distance metric for CBIR system. In [5] and [8], several distance metrics were suggested for both content and texture image retrieval, respectively. In this paper, we are using a
Neutrosophic version of the Euclidean distance, which was presented in [18]. For any two Neutrosophic Sets,

$$
\begin{aligned}
& A=\left\{\left(T_{A}(x), I_{A}(x), F_{A}(x)\right), x \in U\right\} \text { and } \\
& B=\left\{\left(T_{B}(x), I_{B}(x), F_{B}(x)\right), x \in U\right\} \mathrm{in} \\
& U=\left\{u_{1}, u_{2}, u_{3}, \ldots, u_{n}\right\} \text { then }
\end{aligned}
$$

The Neutrosophic Euclidean distance is equal to

$d(A, B)=$

$\sqrt{\sum_{i=1}^{n}\left(\left(T_{A}\left(x_{i}\right)-T_{B}\left(x_{i}\right)\right)^{2}+\left(I_{A}\left(x_{i}\right)-I_{B}\left(x_{i}\right)\right)^{2}+\left(F_{A}\left(x_{i}\right)-F_{B}\left(x_{i}\right)\right)^{2}\right)}$

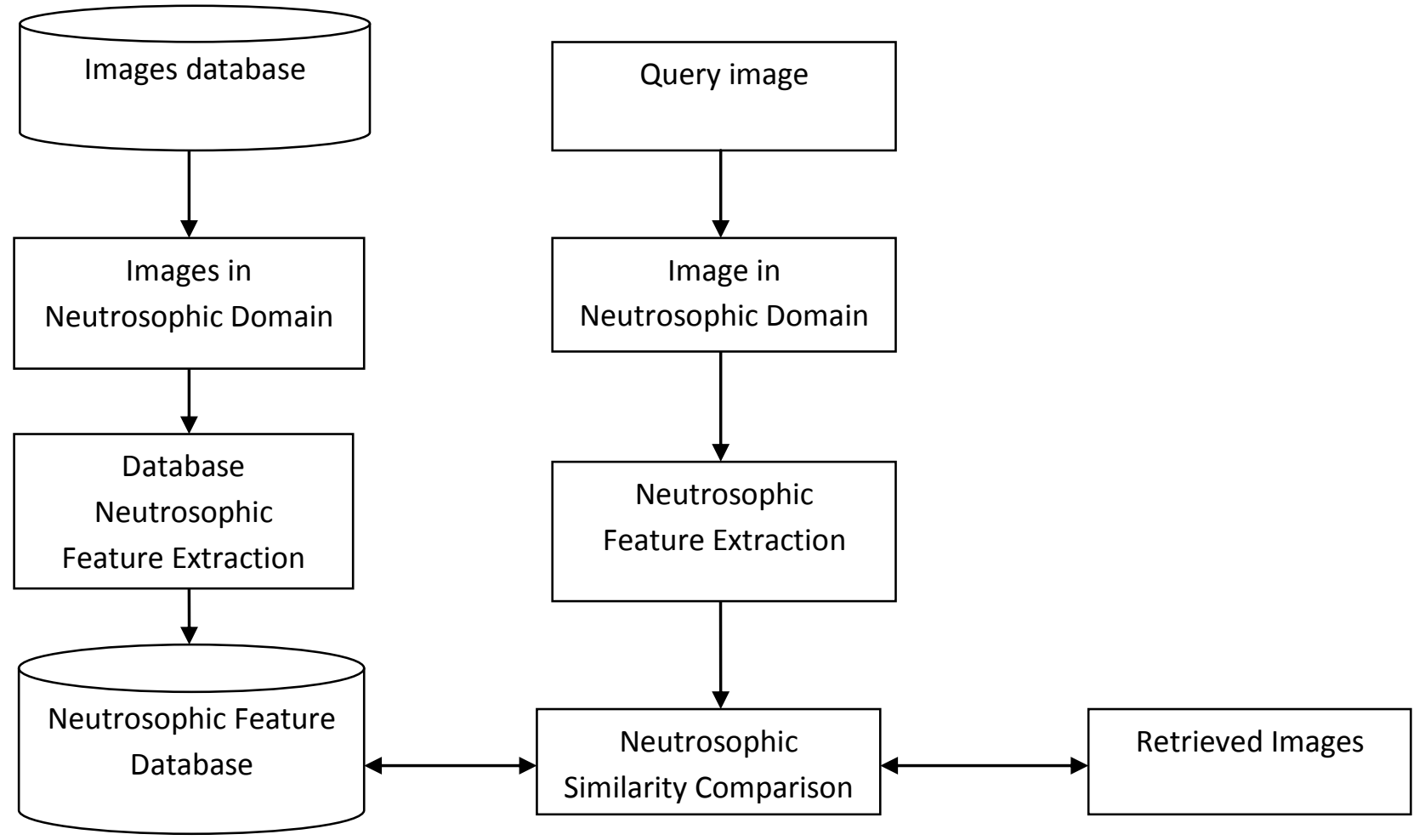

Fig 1: Neutrosophic Image Retrieval Classifier Architecture.

\section{PROPOSED METHOD}

The algorithm for the proposed system is given below which presented in Fig 1:

\section{Algorithm}

STEP1: Convert each image in the database from spatial domain to Neutrosophic domain.

STEP 2: Create a database containing various images.

STEP 3:Extract Texture feature of each image in the database.

STEP 4: Construct a combined feature vector for T, I, F and Stored in another database called Featured Database.

STEP 5: Find the distance between feature vectors of query images and that of featured databases.

STEP 6: Sort the distance and Retrieve the N-top most similar images.

\section{EXPERIMENTAL RESULTS}

The technique which based on NS image retrieval is proposed. First, the image is transformed into NS based on T, I, F and extract neutrosophic texture feature to construct aneutrosophic featured database, which contain the neutrosophicentropy, neutrosophic energy, neutrosophic contrast and neutrosophic homogeneity. NS image retrieval is used to improve the quality and makes retrieval system easier and more effective. The proposed approach was tested on corel dataset. There are 1000 images which contain 10 categories; every category contains 100 images of size $128 \times 58$ in JPG format and compared the performance with that of some existing methods such as image retrieval system based on fuzzy sets. In Fig.2the results of the proposed approach are introduced. The image is transformed from image domain in Fig.2(a) to $\mathrm{T}$ domain objects is seen in Fig.2(b),the Indeterminacy domain objects of the image show in Fig.2(d), Fig.2(d) introduce the image transformed into False domain objects. 


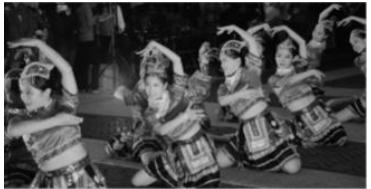

(a)

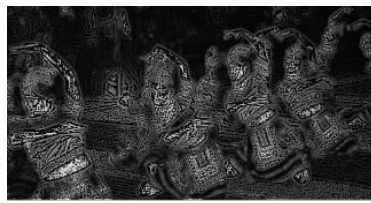

(c)

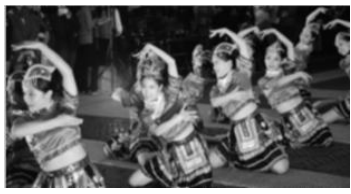

(b)

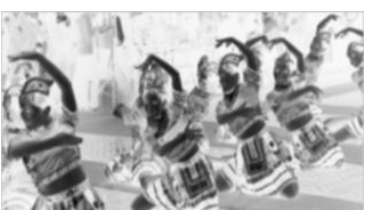

(d)
Fig.2: Image in Neutrosophic domain T, I and F.

In Fig.3 Experiments were introduced using an image database consisting of Corel Image Gallery images. five images selected in the database were used to make query images and their results are categorized by image classes based on the query images using neutrosophic Euclidean distance which used to measure the distance between query image and each image in the database and sort the distances to retrieve the most similar images to the query image.

\subsection{Retrieval Efficiency}

For retrieval efficiency calculation, traditional measures namely precision and recall were calculated using MATLAB workspace database. Precision and Recall [16]:

\section{PRECISION}

$=\frac{\text { NO.OF REVELENT IMAGES RETRIEVED }}{\text { TOTAL NO.OF IMAGES RETRIEVED }}$

\section{RECALL}

\section{NO.OF REVELENT IMAGES RETRIEVED TOTAL NO.OF RELEVENT IMAGES IN THE DATABASE}

We select randomly some sample query images from the database using MATLAB, the system has been tested and the results of the system are shown in Table 1.

Table 1. Precision and Recall value.

\begin{tabular}{|c|c|c|}
\hline Query image & Precision \% & Recall \% \\
\hline (e) & 63 & 5 \\
\hline (f) & 75 & 6 \\
\hline (g) & 87.5 & 7 \\
\hline (h) & 87.5 & 7 \\
\hline (i) & 100 & 8 \\
\hline
\end{tabular}

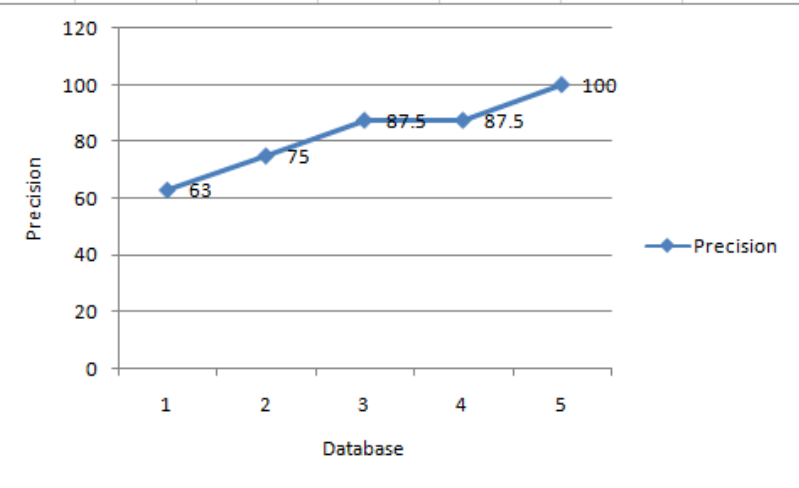

Fig 4: represent the Precision values of the database.

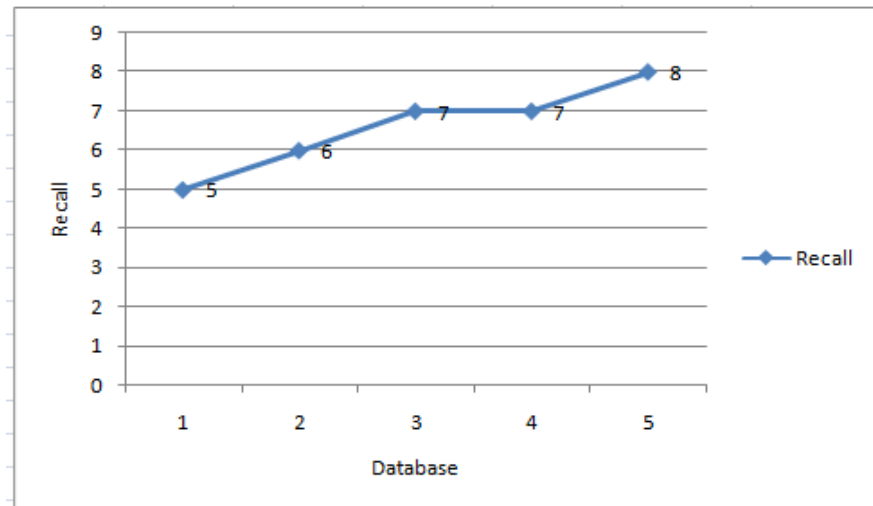

Fig 5: represent the Recall values of the database.

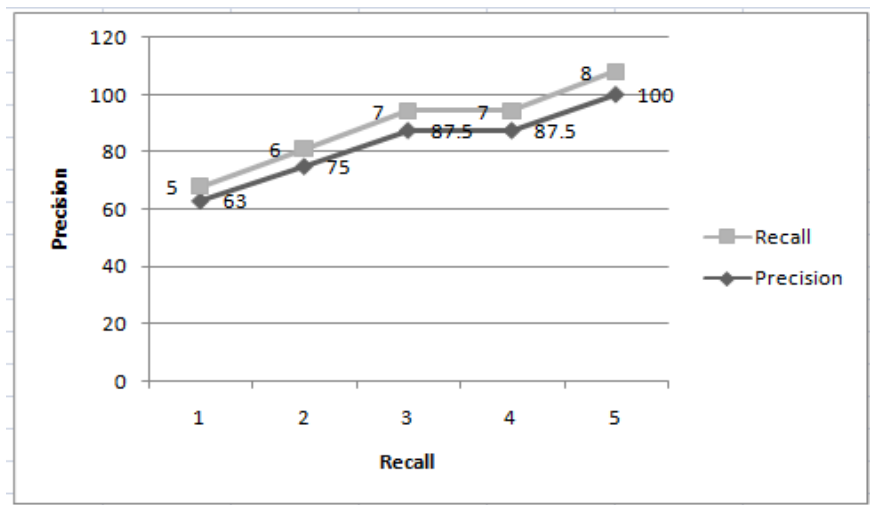

Fig 6: represent the relation between the Precision and Recall values of the database.

\section{CONCLUSION}

In this work, we introduced a new technique for CBIR image retrieval system using Neutrosophic sets which gives better results than image retrieval system based on fuzzy sets which represent in [15]. The proposed work converts each image in the database to Neutrosophic domain. Then, extract texture feature extraction and construct a combined feature vector. Using Euclidean distance between the query image and each image in the database to get the most similar images can be retrieved.

Query Image

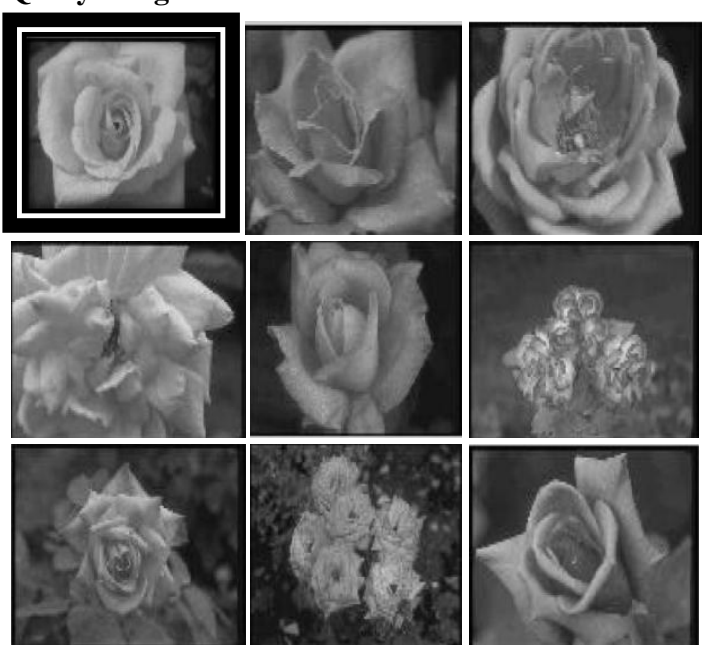

(e) 5 out of 8 


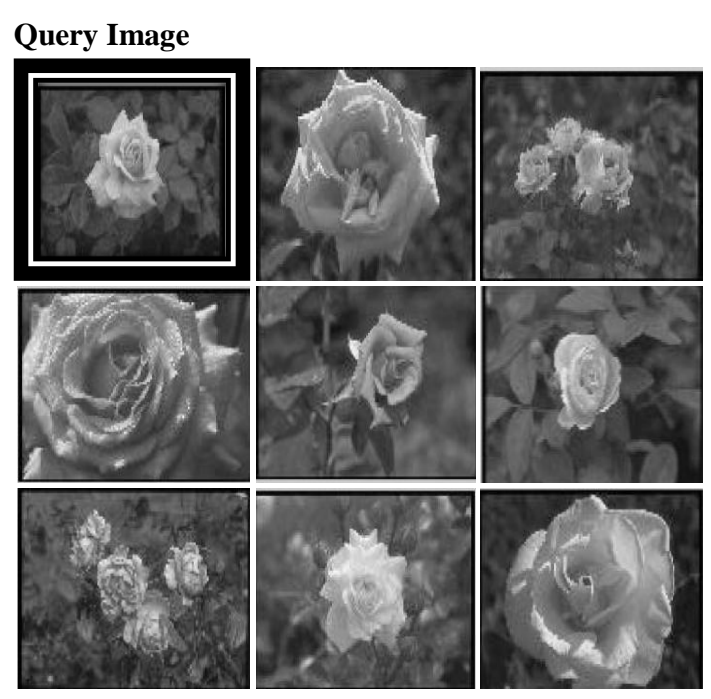

(f) 6 out of 8

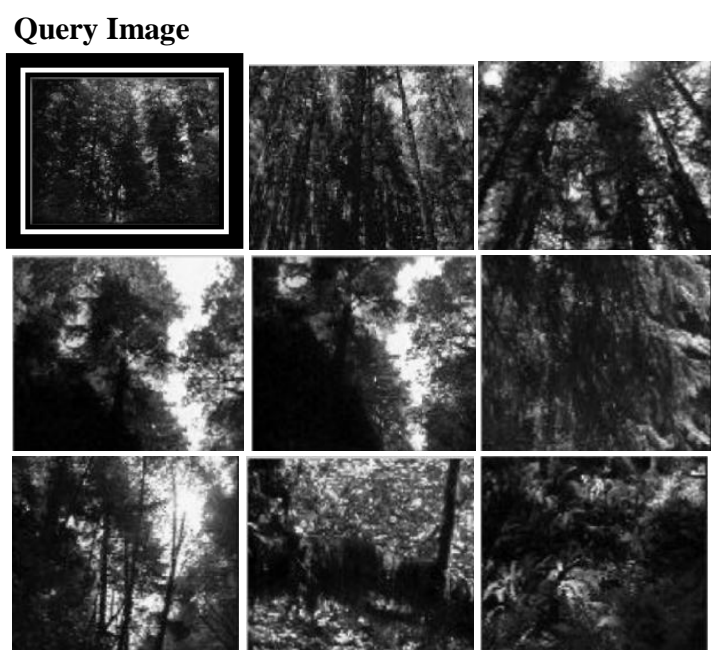

(h) 7 out of 8

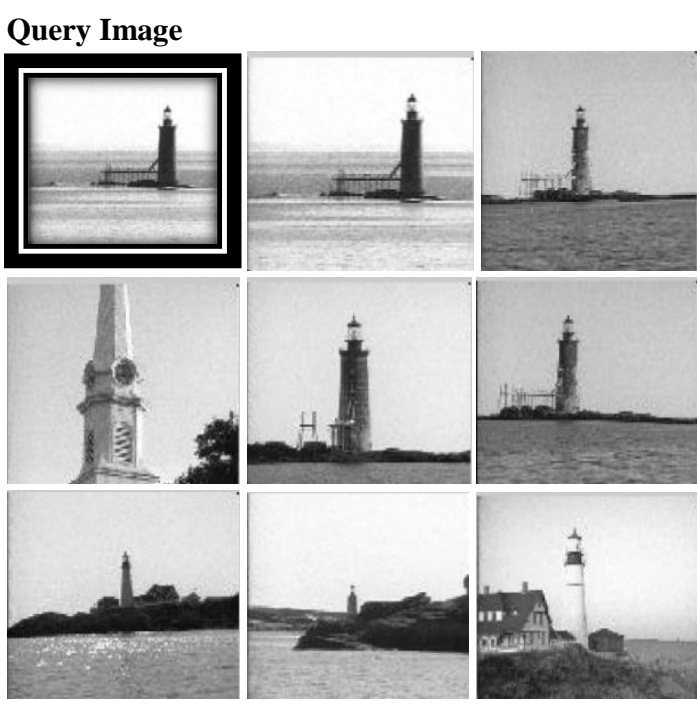

(g) 7 out of 8

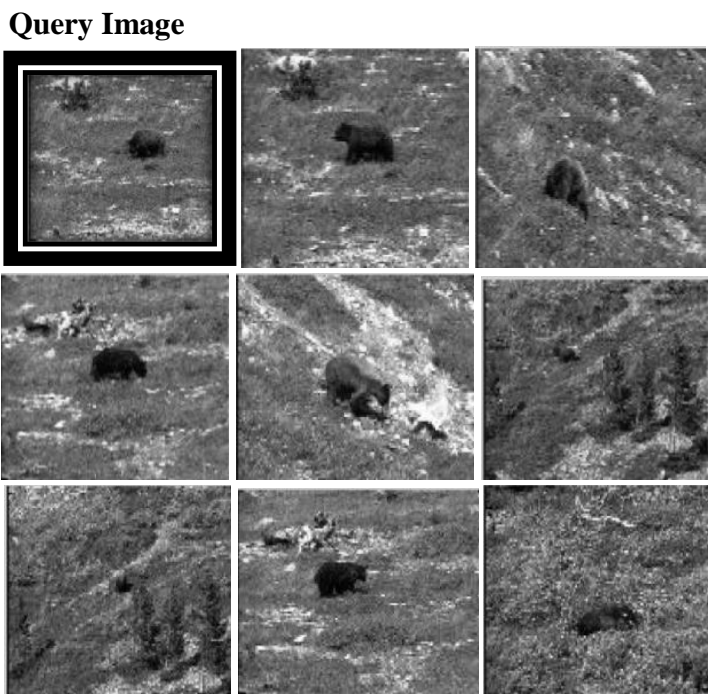

(i) 8 out of 8

Fig.3:The query images and the most similar images which retrieved from the database

\section{REFERENCES}

[1] Chan Y.K. and Chen C.Y., Image Retrieval System Based on Color Complexity and Color Spatial Features, J. of Systems and Software, 71(1), 65-70, 2004.

[2] Cheng H. D., Guot Y., Zhang Y., A Novel Image Segmentation Approach Based on Neutrosophic Set And Improved Fuzzy C- Means Algorithm, World Scientific Publishing Company, New Math. And Natural Computation, 7(1), 155-171, 2011.

[3] Datta, R., Li, J., Wang, J.Z.: Content-Based Image Retrieval: Approaches and Trends of the New Age. Multimedia Information Retrieval, In Proceedings of the 7th ACM SIGMM international workshop on Multimedia information retrieval ACM, 253-262, 2005.

[4] Eisa M., A New Approach For Enhancing Image Retrieval Using Neutrosophic Set, International Journal of Computer Applications, 95(8), 0975-8887, June 2014.
[5] Hanafy I., Salama A.A. and Mahfouz K., Correlation of Neutrosophic Data, in International Refereed Journal of Engineering and Science (IRJES),1(2), 39-43, 2012.

[6] Hanafy I. M., Salama A. A. and Mahfouz K. M., Neutrosophic Classical Events and Its Probability, International Journal of Mathematics and Computer Applications Research (IJMCAR), 3(1), 171-178, mar 2013.

[7] Hearn D. and Baker M. P., Computer Graphics, Englewood Cliffs, NJ: Prentice Hall, ch. 14, 500-504, 1994.

[8] Ingle D., Bhatia Sh., Content Based Image Retrieval using Combined Features, International Journal of Computer Applications , 44(17), 0975-8887, April 2012.

[9] Ionescu, B., Lambert, P., Coquin, D., Buzuloiu, V.: Color based content retrieval of animation movies: a study. In: Proceedings of the International Workshop on 
Content- Based Multimedia Indexing (CBMI 2007), Talence, France, 295-302, June 2007.

[10] Kong F. H., Image Retrieval using Both Color And Texture Features, Department of Information science \& Technology, Heilongjiang Proceedings of the Eighth International Conference on Machine learning and Cybernetics, Baoding, 4, 2228-2232, 12-15 July 2009.

[11] Kuijk A. A. M., Advanced in Computer Graphics Hardware III, Springer,1991.

[12] Lee I., Muneesawang P., Guan L., Automatic Relevance Feedback for Distributed Content-Based Image Retrieval, ICGT, IEEE.org FLEX Chip signal processor ( MC68175/D), Motorola, 1996.

[13] Lew, M.S., Sebe, N., Djeraba, C., Jain, R.: Content-based multimedia information retrieval: State of the art and challenges. ACM Transactions on Multimedia Computing, Communications, and Applications 2(1), 1$19,2006$.

[14] Ma, W.Y., Zhang, H.J.: Content-Based Image Indexing and Retrieval. In: Handbook of Multimedia Computing, CRC Press, Boca Raton, 227-254, 1999.

[15] Puviarasan N., Bhavani R., Vasanthi A., Image Retrieval Using Combination of Texture and Shape Features, 3(3), 5873-5877, March 2014.

[16] Ramamurthy, B., et al. "CBMIR: Content Based Medical Image Retrieval System Using Texture and Intensity for Dental Images." Eco-friendly Computing and Communication Systems. Springer Berlin Heidelberg, 125-134, 2012.

[17] Rui, Y., Huang, T.S., Chang, S.-F.: Image retrieval: current techniques, promising directions, and open issues. Journal of Visual Communication and Image Representation 10(1), 39-62, 1999.

[18] Salama A. A., Abdelfattah M., Eisa M., Distances, Hesitancy Degree and Flexible Querying via Neutrosophic Sets, International of Computer Applications, 101(10), 0975-8887, September 2014.
[19] Salama A. A., Neutrosophic Crisp Point \&Neutrosophic Crisp Ideals, Neutrosophic Sets and Systems, 1(1),50$54,2013$.

[20] Salama A. A. and Smarandache F., Filters Via Neutrosophic Crisp Sets, Neutrosophic Sets and Systems, 1(1), 34-38, 2013.

[21] Salama A. A. and Elagamy H., NeutrosophicFillters, International Journal of Compter Science Engineering and Internation Technology Research (IJCSEITR), 3(1), 307-312, 2013.

[22] Salama A. A., The Concept of Neutrosphic Set and Basic Properties of Neutrosophic Set Operations, WASET 2012 PARIS, FRANC., International university of Science, Engineering and technology, 2012.

[23] Shannon C. E., A Mathematical Theory of Communication, The Bell System Technical Journal, 27, 379-423, 623-656, Jully, October, 1948.

[24] Sinha M. N., Udai A. D., Computer Graphics, Taha McGraw - Hill publishing company limited.

[25] Smarandache F., Neutrosophy and Neutrosophic Logic, First International Conference on Neutrosophy, Neutrosophic logic, Set, Probability, and Statistics, University of New Mexico, Gallup, NM 87301, USA, 2002.

[26] Smarandache F., A Unifying Field in Logics: Neutrosophic Logic. Neutrosophy, Neutrosophic Set, Neutrosophic Probability. Ameican Research Press, Rehoboth, NM, 1-41, 1999.

[27] Smarandache F., Neutrosophic Set, A Generalization of The Intuitionistic Fuzzy Sets, Inter. J. Pure Appl. Math., 24, 287-297, 2005.

[28] Smarandache F., Introduction to Neutrosophic Measure, Neutrosophic Integral and Neutrosophic Probability 2013. 